\title{
Group Contribution-based LCA models to enable screening for environmentally benign novel chemicals in CAMD applications
}

\author{
Pantelis Baxevanidis ${ }^{1}$, Stavros Papadokonstantakis ${ }^{2}$, Antonis Kokossis ${ }^{1}$, and Effie \\ Marcoulaki $^{3}$ \\ ${ }^{1}$ National Technical University of Athens School of Chemical Engineering \\ ${ }^{2}$ Chalmers University of Technology \\ ${ }^{3}$ NCSR Demokritos
}

May 26, 2021

\begin{abstract}
This study considers the development of suitable models for the estimation of Life Cycle Assessment (LCA) indices of organic chemicals based on their molecular structure. The models developed here follow the well-established Group-Contribution (GC) approach and a variety of regression and non-regression methodologies are recruited to achieve the optimum correlation. These models can then be used, alongside other GC models, to screen for molecules with optimal and/or desirable properties, using appropriate molecular design synthesis algorithms. The LCA indices considered here are the Global Warming Potential (GWP), Cumulative Energy Demand (CED) and EcoIndicator 99 (EI99). The model development uses data from existing LCA databases, where each material is associated with its cradle-to-gate LCA metrics, GWP, CED and EI99. The paper presents the model development results, and applies the proposed LCA models on a typical case study for the design of LL-extraction solvents to separate an n-butanol - water mixture.
\end{abstract}

\section{Hosted file}

Pantelis_journal_2021-05-19_TEXT.docx available at https://authorea.com/users/415944/ articles/523699-group-contribution-based-lca-models-to-enable-screening-forenvironmentally-benign-novel-chemicals-in-camd-applications

Hosted file

Pantelis_journal_2021-04-22_TABLES.docx available at https://authorea.com/users/ 415944/articles/523699-group-contribution-based-lca-models-to-enable-screening-forenvironmentally-benign-novel-chemicals-in-camd-applications

\section{Hosted file}

Pantelis_journal_2021-04-22_FIGURES.docx available at https://authorea.com/users/ 415944/articles/523699-group-contribution-based-lca-models-to-enable-screening-forenvironmentally-benign-novel-chemicals-in-camd-applications 